\title{
Maser emission towards the young planetary nebula IRAS 17347-3139
}

\author{
D. Tafoya ${ }^{1,2}$, Y. Gómez ${ }^{2}$, J. F. Gómez ${ }^{3}$, I. de Gregorio-Monsalvo ${ }^{3}$, \\ L. Uscanga ${ }^{2}$, G. Anglada ${ }^{4}$ and J. M. Torelles ${ }^{5}$ \\ ${ }^{1}$ Harvard-Smithsonian Center for Astrophysics, 60 Garden Street, \\ Cambridge, MA 02138, USA \\ ${ }^{2}$ Centro de Radioastronomía y Astrofísica, UNAM Apartado Postal \\ 3-72 (Xangari), 58089 Morelia, Michoacán, México \\ ${ }^{3}$ Laboratorio de Astrofísica Espacial y Física Fundamental INTA, Spain \\ ${ }^{4}$ Instituto de Astrofísica de Andalucía, CSIC Apartado 3004, E-18080, Granada, Spain \\ ${ }^{5}$ Instituto de Ciencias del Espacio, Consejo Superior de Investigaciones Científicas-Instit \\ D'Estudis Espacials de Catalunya, (CSIC-IEEC), Gran Capità 2, E-08034 Barcelona, Spain
}

\begin{abstract}
We have re-analyzed observations of the water maser emission in IRAS 17347-3139 carried out previously and compared them with new higher angular resolution and more sensitive radio-continuum observations to explain the shift between the position of the peak of the radiocontinuum with respect to the center of the ring-like distribution. From our analysis, we found that the water maser emission is not distributed in a closed ring-like structure, but in what appears to be a segment of an ellipse centered in the position of the peak of the radio-continuum. These results can explain the shift between the radio-continuum and the water maser emission. We also present interferometric observations of the OH maser emission towards IRAS 17347-3139 from which we have found that the $\mathrm{OH}$ maser emission detected previously is not associated with this source. Furthermore, since our observations are more sensitive, we have detected for the first time weak $\mathrm{OH}$ maser emission associated with IRAS 17347-3139.
\end{abstract}

Keywords. planetary nebula: individual (IRAS 17347-3139) — masers — radio continuum: stars

\section{Introduction}

IRAS 17347-3139 is one of the two planetary nebula in which water maser emission is known to be present (de Gregorio-Monsalvo et al. 2004). Their VLA observations revealed that the water maser emission is distributed in what seems to be a ring-like structure perpendicular to the lobes observed in the near-IR images. Interestingly, it was also found that the peak of the radio-continuum emission was located not in the geometrical center of such a distribution but towards one of its edges. One suggestion proposed to explain such displacement with the radio-continuum emission being associated with one source, and the water maser emission with a secondary source. However, due to the lack of angular resolution of the observations, it was not possible to arrive at a definitive conclusion. On the other hand, Zijlstra et al. (1989), and more recently Szymczak \& Gérard (2004), reported the detection of $\mathrm{OH}$ maser emission associated with IRAS 17347-3139. However, since the observations were carried out with a single dish and have low angular resolution, the association of such emission with this source was not completely confirmed. In order to understand how the maser emission is distributed in this source, we decided to carry out higher angular resolution observations. 


\section{Observations and results}

On 2005 January 27, we used the VLA in the hybrid configuration, BnA, to carry out high angular observations of the radio continuum at wavelengths $3.6,1.3$ and $0.7 \mathrm{~cm}$, as well as the four $\mathrm{OH}$ maser transitions towards the planetary nebula IRAS 17347-3139. The angular resolution ranges from $\simeq 3.5$ to $\simeq 0^{\prime} .1$ at $\lambda=18$ and $0.7 \mathrm{~cm}$, respectively. In addition, we retrieved the water maser emission data of this source from the VLA archive to re-analyze them and compare them with our observations.

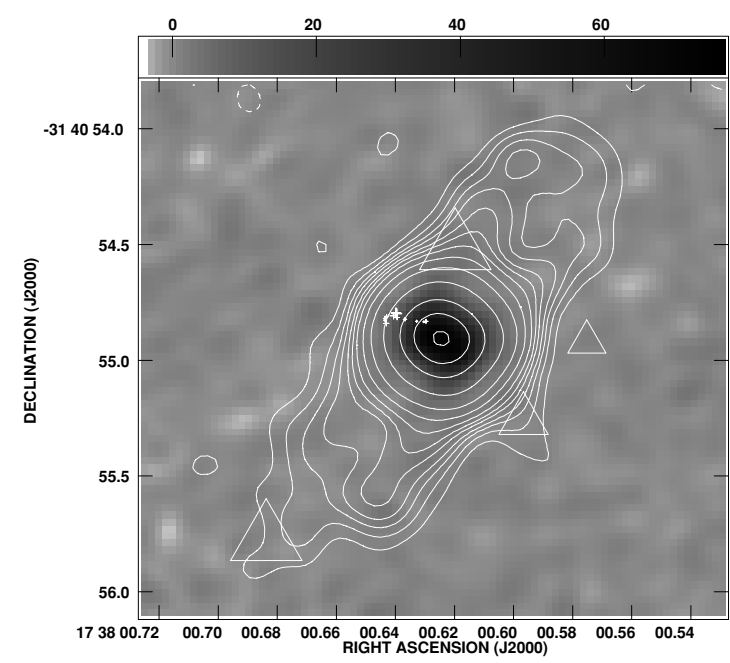

Figure 1. VLA image of IRAS 17347-3139. White contours: $\lambda=1.3 \mathrm{~cm}$, the contours are $-3,3,5$, $7,9,12,15,20,30,50,100,200,300$ and 400 times $2.5 \times 10^{-4} \mathrm{Jy} /$ beam. Grey scale: $\lambda=0.7 \mathrm{~cm}$. For the water and $\mathrm{OH}$ masers, the position of the emission from the different channels are shown in this image as plus signs and triangles, respectively.

In Figure 1, we show an image of the radio-continuum emission of IRAS 17347-3139 at $\lambda=1.3$ and $0.7 \mathrm{~cm}$ in contours and grey scale, respectively. The water maser emission is shown as plus signs towards the north-east of the peak of the continuum emission. From our analysis, we have found that the masers are distributed in what appears to be a segment of an ellipse of size $\simeq 0$ " $1 \times 0 .{ }^{\prime \prime} 5$ centered at the peak of the continuum. This result, along with the image at $\lambda=0.7 \mathrm{~cm}$, suggests that the water maser emission is arising in a circumstellar high density torus-like structure.

Finally, from the four $\mathrm{OH}$ maser transitions, we have detected emission of the $1612 \mathrm{MHz}$ line at three velocity ranges: i) from 5 to $-24 \mathrm{~km} \mathrm{~s}^{-1}$, ii) $\simeq-70 \mathrm{~km} \mathrm{~s}^{-1}$ and iii) from -90 to $-123 \mathrm{~km} \mathrm{~s}^{-1}$. The emission in the first range is located $\simeq 11^{\prime}$ south of the position of IRAS 17347-3139, and it has been associated with the $\mathrm{OH} / \mathrm{IR}$ star $\mathrm{OH} 356.65-0.15$ (Bowers \& Knapp 1989). The emission in the third range is located 2'.4 to the north-east of the position of IRAS 17347-3139, probably too far to be associated to this source. The only emission that coincides with the position of IRAS 17347-3139 is at $\mathrm{v}_{L S R} \simeq$ $-70 \mathrm{~km} \mathrm{~s}^{-1}$, which is detected for the first time with our observations and is shown as triangles in Figure 1.

\section{References}

Bowers P. F. \& Knapp G. R., ApJ, 347, 325

de Gregorio-Monsalvo, I., Gómez, Y., Anglada, G., Cesaroni, R., Miranda, L. F., Gómez, J. F. \& Torrelles, J. M. 2004, ApJ, 601, 921

Szymczak \& Gérard, 2004, A\&A, 423, 209

Zijlstra, A. A., Te Lintel Hekkert, P., Pottasch, S. R., Caswell, J. L., Ratag, M., \& Habing, H. J., 1989, A\&A, 217, 157 